ADVERSE DRUG REACTION

\title{
Acute irritant reaction to an antiseptic bath emollient
}

\author{
N K Saw, J R Hindmarsh
}

Postgrad Med J 2005;81:131-132. doi: 10.1136/pgmj.2003.018564

Antiseptic bath emollients are commonly prescribed for treatment of eczema and are generally safe for frequent application. Although acute irritant reactions are uncommon it is nevertheless recognised and could have significant morbidity. This case describes a young male patient who developed an acute irritant reaction localised to the external genitalia, mimicking Fournier's gangrene, after overnight application of Oilatum Plus antiseptic bath emollients.
A 27 year old immunocompetent man presented with one day history of painful diffuse swelling of the scrotum and penis associated with difficulty to micturate. The symptoms appeared after full body application of Oilatum Plus (active ingredients: benzalkonium chloride 6.0\%, triclosan $2 \%$, light liquid paraffin $52.5 \%$ ) that was prescribed for eczema the previous day. No history of trauma or recent sexual intercourse was elicited.

On examination, he was afebrile but distressed. His pulse rate was 110 beat/min and his blood pressure was 140/ $90 \mathrm{~mm} \mathrm{Hg}$. Examination of the external genitalia showed tender diffuse swelling of the scrotum and penis (fig 1). Urinary bladder was palpable and on scanning confirmed acute urinary retention with a residual of $600 \mathrm{ml}$. Initial differential diagnosis was Fournier's gangrene and treatment with parenteral broad spectrum antibiotics was started. A suprapubic catheter was inserted after failed urethral catheterisation.

Subsequent full blood count, biochemical profile, and cystourethrography were normal while ultrasonography showed pronounced scrotal oedema. Wound swab and urine culture were negative.

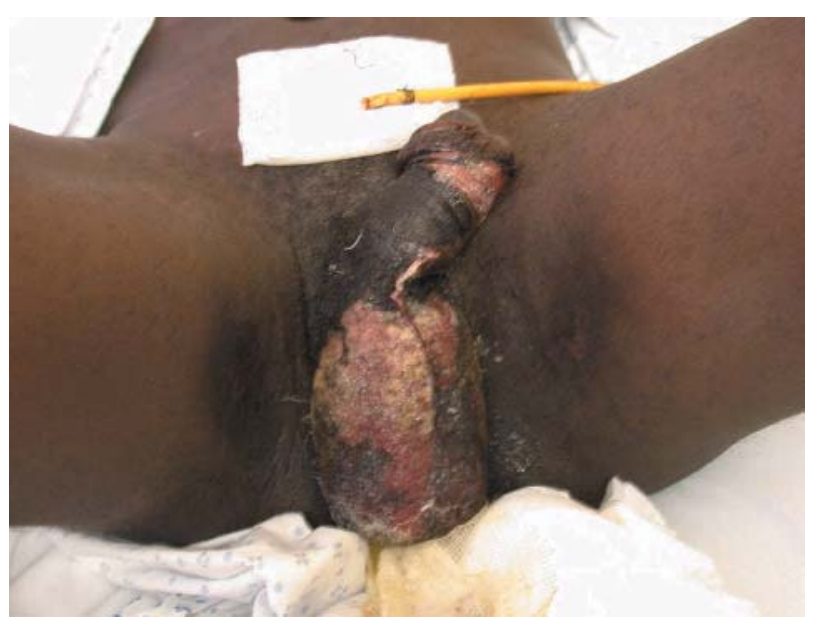

Figure 1 Diffuse scrotal and penile swelling with extensive skin ulceration.

\section{Summary points}

- Antiseptic bath emollients can cause severe acute irritant reaction associated with significant morbidity.

- Male patients should be cautioned regarding application of antiseptic emollients to the external genitalia because of its susceptibility to develop acute irritant reaction.

The rapid recovery after cessation of emollient application suggested an acute irritant reaction as the most probable diagnosis. The patient was managed conservatively and discharged five days later with normal urinary function.

\section{DISCUSSION}

Irritant dermatitis associated with antiseptic bath emollients is rare. Only two reports have been recorded in literature to date; both secondary to application of Oilatum Plus. ${ }^{12}$

The active ingredients in this emollient, triclosan and benzalkonium chloride, have both been reported to cause irritant dermatitis. ${ }^{3}{ }^{4}$ However, benzalkonium chloride is six times more likely to trigger a hypersensitivity reaction than the triclosan and additionally becomes corrosive at a concentration level of $10 \% .{ }^{15}$ The concentration of benzalkonium chloride in Oilatum Plus is noted to be significantly higher than most antiseptic bath emollients, which possibly accounts for the acute irritant reactions. ${ }^{1}$

Although the emollient was applied to the whole body the irritant reaction was confined to the external genitalia because the scrotal skin has remarkable permeability and hence is more susceptible to toxic and irritant agents. ${ }^{6}$

It is important for clinicians to be aware of this adverse drug reaction when prescribing the treatment to a patient, because of the associated morbidity, especially in male patients, who could present with clinical features similar to Fournier's gangrene.

\section{Authors' affiliations \\ N K Saw, J R Hindmarsh, Department of Urology, The James Cook University Hospital, Middlesbrough, UK}

Correspondence to: Mr J R Hindmarsh, Department of Urology, The James Cook University Hospital, Marton Road, Middlesbrough TS4 3BW, UK; john.hindmarsh@stees.nhs.uk

Submitted 4 January 2004

Accepted 27 April 2004

\section{REFERENCES}

1 Loo WJ. Irritant dermatitis due to prolonged contact with Oilatum Plus. Br J Dermatol 2003;148:171-2.

2 Ling TC, Highet AS. Irritant reactions to an antiseptic bath emollient. J Dermatol Treat 2000;11:263-7. 
3 Park $\mathrm{HJ}$, Kang HA, Lee JY, et al. Allergic contact dermatitis from benzalkonium chloride in antifungal solution. Contact Dermatitis 2000;42:306-7.

4 Wong CS, Beck MH. Allergic contact dermatitis from triclosan in antibacterial handwashes. Contact Dermatitis 2001;45:307.
5 Perrenourd D, Bircher A, Hunziker T et al. Frequency of sensitization to 13 common preservatives in Switzerland. Swiss Contact Dermatitis Research Group. Contact Dermatitis 1994;30:276-9.

6 Fisher AA. Unique reactions of scrotal skin to topical agents. Cutis 1989:44:445-7.

FILLER

Words for a curriculum vitae or a reference

was asked to provide examples of words that might feature in a contemporary curriculum vitae or in a reference. Words in bold are, I suggest, attributes, those underlined are less than complimentary, and those in italic are either meaningless or capable of either interpretation. All words suggested are acceptable and unlikely to result in legal action. Context is everything.

- Amiable, academic, astute, adroit, awesome, amazing, accommodating, aristocratic.

- Brilliant, bland

- Capable, conscientious, confident, calculating, circumspect, candid, conventional, candid, creative, conservative

- Dedicated, dependable, diplomatic, dynamic, determined, debonair, decisive, distinctive, distinguished, difficult

- Enthusiastic, effective, erudite, exceptional, equanimity, extraordinary

- Forceful

- Gregarious

- Holistic, humane, high minded

- Imaginative, intelligent, imperturbable, imposing, independent, incisive, intuitive

- Judious

- Keen, knowledgable, knowing

- Liberal, laconic

- Mature

- Original, opinionated

- Politically active, philosophical, penetrating, phenomenal

- Questioning, quick

- Reflective, reliable, rational, resourceful, relaxed

- Scrupulous

- Talented, temperate, tolerant, tireless

- Unassuming unmistakable, unconventional, uncommon, uncompromising, undisputed, unparalleled, unmistakable

- Versatile, venerable

- Willing, wise

- Zealous

P D Welsby Western General Hospital, Edinburgh EH4 2XU, UK; P.Welsby@ed.ac.uk 